\title{
PERFIL EPIDEMIOLOGICO DOS ÓBITOS OCASIONADOS PELA SEPTICEMIA, NA REGIÃO NORDESTE DO BRASIL, ESTADO DO RIO GRANDE DO NORTE - RN
}

\begin{abstract}
Adriano Menino de Macedo Júnior ${ }^{1}$
Josimar Torres Gomes²

\section{Resumo}

Introdução: $\mathrm{O}$ presente artigo mapeia o perfil epidemiológico da sepse no estado do Rio Grande do Norte - RN, favorecendo informações as autoridade em saúde publica, para criação de novos métodos, no combate a sepse, bem como diagnostico precoce e tratamentos mais eficazes. Materiais e métodos: trata-se de um estudo epidemiológico, retrospectivo realizado com a plataforma do DATASUS, referente à Região Nordeste do Brasil - BR, no período de julho de 2019 a abril de 2020. Resultados: A faixa etária mais acometida por óbitos pela sepse é a de 70 a 79 anos de idade, percentuando um total de 22,69\% ( $\mathrm{n}=133$ casos), e na faixa etária maior que 80 anos, registrando $34,98 \%$ ( $n=205$ casos), a maior taxa de mortalidade ocorreu no sexo feminino, representando um percentual de 50,42\% ( $\mathrm{n}=297$ casos), enquanto que no gênero masculino o percentual foi de 49,31\% ( $\mathrm{n}=289$ casos), A sepse entra no capitulo I, Doenças infecciosas e parasitárias, 20,68\% ( $n=1.219$ casos), número que corresponde a homens e mulheres, sendo que desse total de óbitos, 48,07\% $(\mathrm{n}=586)$, foi ocasionado pela sepse. Conclusão: A alta taxa de mortalidade ocasionada pela sepse é preocupante, daí a importância de melhorias na saúde brasileira.
\end{abstract}

Palavras-chave: Sepse, Saúde Público, UTI, mortalidade, perfil epidemiológico.

\begin{abstract}
Abstract: This article maps the epidemiological profile of sepsis in the state of Rio Grande do Norte - RN, favoring information to public health authorities, for the creation of new methods, in the fight against sepsis, as well as early diagnosis and more effective treatments. Materials and methods: this is an epidemiological, retrospective study carried out with the DATASUS platform, referring to the Northeast Region of Brazil - BR, from July 2019 to April 2020. Results: The age group most affected by deaths by sepsis is 70 to 79 years old, with a total percentage of $22.69 \%$ ( $n=133$ cases), and in the age group older than 8o years, registering $34.98 \%$ ( $\mathrm{n}=205$ cases), the highest rate of mortality occurred in females, representing a percentage of $50.42 \%(n=297$ cases), while in males the percentage was $49.31 \%(n$ $=289$ cases). Sepsis enters chapter I, Diseases infectious and parasitic, $20.68 \%$ (n $=1,219$ cases), a number that corresponds to men and women, and of this total of deaths, $48.07 \%(n=586)$, was caused by sepsis. Conclusion: The high mortality

1 Farmacêutico - bioquímico. UNICEUNA - Centro Universitário Natalense. Departamento Farmácia. Natal, Rio Grande do Norte, Brasil. ORCID: https://orcid.org/oooo-ooo1-6367-1088; Lattes: http:// lattes.cnpq.br/4134152465913204. E-mail: adrianomenino2016@gmail.com.

2 Doutorado em produção animal pelo Programa de Doutorado Integrado em Zootecnia da UFPB UFC - UFRPE. UFPB - Universidade Federal da Paraíba. Departamento de Zootecnia. Areia - Paraíba, Brasil. Lattes: http://lattes.cnpq.br/5974891623513289; E-mail: josimartg@yahoo.com.br.
\end{abstract}


rate caused by sepsis is worrying, hence the importance of improvements in Brazilian health.

Keywords: Sepsis, Public Health, ICU, mortality, epidemiological profile.

\section{Resumen}

Introducción: Este artículo mapea el perfil epidemiológico de la sepsis en el estado de Rio Grande do Norte - RN, favoreciendo la información a las autoridades de salud pública, para la creación de nuevos métodos, en la lucha contra la sepsis, así como el diagnóstico precoz y tratamientos más efectivos. Materiales y métodos: este es un estudio epidemiológico retrospectivo realizado con la plataforma DATASUS, que hace referencia a la Región Nordeste de Brasil - BR, desde julio de 2019 hasta abril de 2020. Resultados: el grupo de edad más afectado por las muertes por la sepsis es de 70 a 79 años, con un porcentaje total de $22.69 \%$ ( $n=133$ casos), y en el grupo de edad mayor de 80 años, registrando $34.98 \%$ ( $\mathrm{n}=205$ casos), la tasa más alta la mortalidad se produjo en las mujeres, lo que representa un porcentaje del 50,42\% ( $\mathrm{n}=297$ casos), mientras que en los hombres, el porcentaje fue del 49,31\% ( $n=289$ casos). La sepsis entra en el capítulo I, Enfermedades infeccioso y parasitario, 20.68\% ( $\mathrm{n}=1,219$ casos), un número que corresponde a hombres y mujeres, y de este número total de muertes, 48.07\% ( $\mathrm{n}=586)$, fue causado por sepsis. Conclusión: La alta tasa de mortalidad causada por la sepsis es preocupante, de ahí la importancia de las mejoras en la salud brasileña.

Palabras clave: sepsis, salud pública, UCI, mortalidad, perfil epidemiológico.

\section{INTRODUÇÃO}

Desde os primórdios dos tempos, múltiplas infecções acompanham a evolução do ser humano, afetando a saúde com invasões de microrganismos com potencias patógenos, em muitos casos com virulência capaz de causa a morte dos indivíduos. Nesse processo de invasão dos microrganismos nos hospedeiros desencadeiam diversas respostas imunológicas, para reestabelecer a saúde do corpo. Se o organismo humano não for capaz de controlar a infecção ou resposta inflamatória exagerada, respectivamente, com seu sistema imunológico ou com a homeostasia, o quadro pode se agravar e chegar a uma sepse. (WEBER, 2019; RIBEIRO \& PIRES, 2018).

A sepse é uma disfunção que pode ocorrer em todo o corpo humano, comprometendo a vida do paciente, ocasionada pela desregulação da resposta imune do hospedeiro a determinadas infecções. (MENEZES et al., 2019; LUZ FILHO et al., 2019).

Segundo Luiz Filho et al. (2019), a sepse ocorre através do conjuntos de reações inflamatórias, neurais, hormonais e metabólicas, que caracterizam a Síndrome Inflamatória Sistêmica (SIRS), que é o resultado das manifestações inter-relacionados entre microrganismos patógenos e a resposta imunológica do hospedeiro. A SIRS, 
pode se agravar em sepse, sepse grave e choque séptico, o que elevam as altas taxas de mortalidade existentes no país.

Em 1992, foi realizada a primeira conferência a respeito da Sepse, onde à mesma ficou determinada como Síndrome de Resposta Inflamatória Sistêmica (SIRS) frente processo infeccioso confirmado ou suspeito. (ALMEIDA et al., p. 7, 2018).

[...] Neste mesmo consenso, outras definições foram estabelecidas. SIRS passou a ser determinada pela presença de, no mínimo, dois dos seguintes sinais: temperatura central $>38,3^{\circ} \mathrm{C}$ ou $<36^{\circ} \mathrm{C}$; frequência cardíaca $>90 \mathrm{bpm}$, frequência respiratória $>20$ irpm ou $\mathrm{PaCO}_{2}<32 \mathrm{mmHg}$ e leucócitos totais $>12.000 / \mathrm{mm}^{3}$ ou $<4.000 / \mathrm{mm}^{3}$. Sepse grave ficou definida como sinais de sepse associados à disfunção orgânica e/ou hipoperfusão tecidual. Por fim, determinou-se choque séptico como falência circulatória aguda, caracterizada por hipotensão arterial persistente em paciente séptico. (ALMEIDA et al., p. 7, 2018.)

A partir então do ano de 2001, uma nova conferencia foi realizada, com o objetivo de ampliar os critérios para o diagnostico da Sepse, buscando em seu diagnostico clínico, sintomas suspeitos como: febre e hipotermia, taquicardia, taquipneia, alteração do exame neurológico, hiperglicemia, edema; e no diagnostico laboratorial, processos inflamatórios como: leucocitose, leucopenia, elevação de procalcitonina ou de proteína $C$ reativa; outras hemodinâmicas como: hipotensão arterial, saturação venosa baixa e débito cardíaco aumentado. (ALMEIDA et al., 2018; ROCHA et al., 2019).

Ainda nessa conferencia foi elaborado o PIRO (Predisposição, Infecção, Resposta, Disfunção Orgânica), que visa avaliar o risco e predizer desfechos em pacientes sépticos, bem como auxiliar a inclusão desses pacientes em estudos clínicos, classificando-os em estágios semelhantes em termos prognósticos e da viabilidade de responder a terapias medicamentosas específicas. Em 2016, surgiu um novo consenso sobre a sepse, está não estava mais ligada apenas a um agente infeccioso como o microrganismo, e sim a processos inflamatórios, e anti-inflamatórias, desencadeado por, mal funcionamento, do próprio organismo do hospedeiro. (WEBER, 2019; JOST et al., 2019).

Durante o Terceiro Consenso Internacional de Definições para a Sepse e Choque Séptico no ano de 2016, sugeriu-se a adoção de um novo score, para analise de cuidados críticos sobre Sepse, dispondo do SOFA (Classificação Sequencial da Avaliação de Falência de Órgãos), Critério para a Síndrome da Resposta Inflamatória Sistêmica (SIRS), Sistema Lógico de Disfunção de Órgãos (LODS). Para chegar a uma maneira mais rápida de detectar nos órgãos do corpo humano, a sepse, foi criado então, a Avaliação Rápida Sequencial da Falência de Órgãos (qSOFA). (WEBER, 2019). 
O método de SOFA apresenta divergências na agilidade do diagnóstico do paciente, fora das Unidades de Terapias Intensivas, devido a sua complexidade, colocando em risco a vida do individuo. A pontuação do SOFA marca uma escala de o a 4 pontos, onde são analisados à respiração, coagulação, função hepática e função cardiovascular dos pacientes, e assim, a maior pontuação evidencia a probabilidade de morte do paciente. (WEBER, 2019).

Já o método qSOFA, adere a pontuação na escala de o a 3, seguindo os critérios de hipotensão (pressão sistólica abaixo de 10ommHg), alterações no estado mental, e taquipneia (frequência respiratória maior que irpm). (WEBER, 2019).

Embora o sangue normalmente seja estéril, números moderados de micro-organismos podem entrar na corrente sanguínea sem causar dano. Em condições hospitalares, o sangue muitas vezes é contaminado como resultado de procedimentos invasivos, como a inserção de cateteres e tubos de alimentação intravenosa. O sangue e a linfa contêm várias células fagocíticas defensoras. Além disso, o sangue tem pouco ferro disponível, que é necessário para o crescimento bacteriano. Entretanto, se as defesas dos sistemas cardiovascular e linfático falham, os micróbios podem proliferar no sangue. Uma doença aguda que está associada com a presença e a persistência de micro-organismos patogênicos ou suas toxinas é denominada septicemia. Um termo similar que não é equiparado do ponto de vista médico com a septicemia é sepse, embora haja uma tendência em usá-los como sinônimos. A sepse é definida como uma síndrome da resposta inflamatória sistêmica (SIRS, de systemic inflammatory response syndrome) causada por um foco de infecção que libera mediadores da inflamação dentro da corrente sanguínea. O local de infecção em si não é necessariamente a corrente sanguínea, e em cerca de metade dos casos nenhum micróbio é encontrado no sangue. A SIRS deve exibir pelo menos duas de um grupo de condições definidas: febre, taxas cardíaca ou respiratória rápidas e uma alta contagem de células brancas do sangue. Se as bactérias infectivas provocarem a lise das hemácias, a liberação de hemoglobina contendo ferro pode resultar em aceleração do crescimento microbiano. A sepse e a septicemia geralmente são acompanhadas pelo aparecimento de linfangite, vasos linfáticos inflamados visíveis como estrias vermelhas sob a pele, correndo ao longo do braço ou da perna a partir do local da infecção. (TORTORA et al., p. 639, 2012).

As Unidades de Terapias Intensivas, lida com desafios diários, por se tratar de uma ala que cuida de pacientes em estado grave, adquiridos por meio de traumas ou pós-operatórios, doenças e infecções adquiridas, que possam resultar no óbito imediato do paciente. A UTI é composta por uma equipe de multiprofissionais, que visam à assistência do paciente, 24 horas por dia, garantindo eficácia do tratamento 
e recuperação do paciente. A sepse é um problema grave de saúde pública e pode ser fatal para o paciente, pois sua presença hoje está mais registrada nas UTIs, onde os pacientes já estão fragilizados e em recuperação, e a dificuldade que os profissionais têm em identificar a infecção por Sepse, bem como o tratamento que deve ser minucioso que exige agilidade e precisão no momento do diagnostico. (Seibt et al., 2019; Patricio e Domingues, 2019).

A sepse é considerada a principal causa de morte em unidade de terapia intensiva (UTI). Entre 6 e 54\% dos pacientes internados neste setor tem sepse grave e nesses casos a mortalidade é de 20 a 60\%. Além disso, incorre em longo período de internação e consequentemente elevados custos para o sistema de saúde. As complicações podem ocasionar ainda danos irreversíveis ao indivíduo acometido, uma vez que a possibilidade de novas infecções nessas pessoas é de três a cinco vezes maior que em indivíduos que nunca tiveram sepse, além da incidência de tumores. E ainda alguns estudo destacam que a expectativa de vida é menor. A sepse é uma das condições mais frequentes em todo o mundo para as quais o cuidado da UTI é requerido. (ZONTA et al., p. 1, 2018).

A sepse quando ocorre em neonatos, à infecção pode agravar-se sistematicamente no bebê por volta das 72 horas de vida, a infecção pode ser detectada pelas alterações clínicas ou laboratoriais. Muitos fatores podem ocasionar a doença, nos recém-nascido, como a contaminação por bactérias gram-positivas e gram-negativas, bem como outros riscos pertinentes ao caso, como baixo peso do recém-nascido, tipo de parto, ambos podem contaminar o bebê com patógenos, entre outros. A Sepse em recém-nascidos e umas das principais causas perinatais que ocorre no primeiro mês de vida da criança, ficando atrás somente das doenças pulmonares e das doenças congênitas. (SANTOS et al., 2018; SILVA et al., 2018).

Este trabalho tem o objetivo de alertar as autoridades públicas para o grave problema de Saúde Pública, ocasionado pela sepse, uma condição que causa na maioria das vezes a morte do paciente, o trabalho enfatiza a importância do diagnostico precoce da sepse, para reduzir as altas taxas de mortalidade, não só no estado do Rio Grande do Norte - RN, mas para o mundo todo, pois sepse é uma condição que afeta todas as pessoas.

\section{METODOLOGIA}

Está é uma pesquisa transversal, desenvolvida na forma descritiva e retrospectiva, de natureza epidemiológica, com dados fornecido e extraídos da base 
de dados do Sistema Nacional de Agravos de Notificação (SINAN), estatísticas essas disponibilizadas pelo DATASUS.

Os dados foram filtrados na plataforma pelo período de julho de 2019 a abril de 2020, para os casos de óbitos confirmados, no Estado do Rio Grande do Norte RN, no Brasil - BR, país integrante da América do Sul, a pesquisa especifica ocorre na Região Nordeste do país.

As variáveis avaliadas nos resultados foram: óbitos de acordo com ano e mês, segundo a Faixa Etária; óbitos de acordo com ano e mês, segundo o sexo dos pacientes; e óbitos por ano e mês, óbitos por gênero segundo capítulo do CID-1o e óbitos por mês/ano, segundo o capitulo CID-10: I, algumas doenças infecciosas e parasitárias, lista de morbidade CID-10: Septicemia.

Todos os dados epidemiológicos coletados para análise foram selecionados e obtidos por meio do aplicativo TABNET, a partir de suas caixas de opções (linha, coluna e conteúdo). Por se tratar de dados secundários de um banco de domínio público, não foi necessário submeter o trabalho ao Comitê de Ética em Pesquisa em Seres Humanos (CEP).

A metodologia da presente pesquisa utilizou também pesquisas bibliográficas, como revisão de literatura, a qual foi lastreada em artigos constantes na base eletrônica SciELO, Google acadêmico, Pubmed, MEDLINE (National Library of Medicine, USA - NLM), assim como repositórios de universidades públicas e privadas, empregando-se as palavras chave: perfil epidemiológico da Sepse, septicemia, Saúde Pública.

A pesquisa considerou artigos publicados entre os anos de 2018 e 2020 , de forma que se realizou, inicialmente, a seleção, por vias das palavras chaves. Em seguida, promoveu-se o fichamento do material selecionado e elegeram-se os artigos que faziam alusão ao tema. Os critérios de inclusão foram: artigos correlatos ao tema, publicados entre 2018 e 2020 . Os critérios de exclusão foram: artigos concernentes ao tema, entretanto, dentro da área Saúde Pública.

\section{RESULTADOS E DISCUSSÃO}

No período de julho/2019 até abril/2020, foram notificados elevados números de óbitos, na Região Nordeste do Brasil, especificamente o Rio Grande do Norte RN, na tabela numero 1, podemos ver o grau de acometimento da sepse, segundo a faixa etária. 
Tabela 1. Óbitos de acordo com ano e mês, segundo a Faixa Etária:

\begin{tabular}{|c|c|c|c|c|c|c|c|c|c|c|c|}
\hline Faixa etária & $\begin{array}{l}2019 \\
\text { /Jul. }\end{array}$ & $\begin{array}{l}\text { 2019/ } \\
\text { Ago. }\end{array}$ & $\begin{array}{l}2019 / \\
\text { Set. }\end{array}$ & $\begin{array}{l}2019 / \\
\text { Out. }\end{array}$ & $\begin{array}{l}2019 / \\
\text { Nov. }\end{array}$ & $\begin{array}{l}\text { 2019/ } \\
\text { Dez. }\end{array}$ & $\begin{array}{l}2020 / \\
\text { Jan. }\end{array}$ & $\begin{array}{c}2020 / \\
\text { Fev. }\end{array}$ & $\begin{array}{l}2020 / \\
\text { Mar. }\end{array}$ & $\begin{array}{c}2020 / \\
\text { Abr. }\end{array}$ & Total \\
\hline $\begin{array}{l}\text { Menor de um } \\
\text { ano }\end{array}$ & 3 & 4 & 3 & - & - & 1 & 2 & 3 & 5 & 1 & 22 \\
\hline 1 a 4 anos & 1 & - & - & 1 & 1 & - & 1 & 1 & 1 & 1 & 7 \\
\hline 5 a 9 anos & 1 & 1 & - & - & - & 1 & 1 & 2 & 1 & 1 & 8 \\
\hline 10 a 14 anos & 1 & - & - & - & - & - & - & - & - & 2 & 3 \\
\hline 15 a 19 anos & - & - & - & - & - & 1 & - & - & - & 1 & 2 \\
\hline 20 a 29 anos & 2 & - & 1 & 2 & - & - & 2 & 1 & 4 & 1 & 13 \\
\hline 30 a 39 anos & 5 & 2 & 1 & 1 & - & 4 & - & 4 & 3 & 2 & 22 \\
\hline 40 a 49 anos & 5 & 2 & 4 & 4 & 3 & - & 2 & 2 & 1 & 2 & 25 \\
\hline 50 a 59 anos & 8 & 6 & 6 & 7 & 3 & 2 & 11 & 4 & 6 & 7 & 60 \\
\hline 60 a 69 anos & 15 & 11 & 11 & 8 & 6 & 6 & 6 & 9 & 7 & 7 & 86 \\
\hline 70 a 79 anos & 15 & 6 & 11 & 17 & 17 & 16 & 13 & 11 & 10 & 17 & 133 \\
\hline$>80$ anos & 25 & 11 & 19 & 22 & 34 & 14 & 21 & 16 & 23 & 20 & 205 \\
\hline Total & 81 & 43 & 56 & 62 & 64 & 45 & 59 & 53 & 61 & 62 & 586 \\
\hline
\end{tabular}

Fonte: DATASUS, 2020.

Podemos ver no presente estudo que a faixa etária mais acometida por óbitos pela sepse é a de 70 a 79 anos de idade, percentuando um total de 22,69\% ( $n=133$ casos), e na faixa etária maior que 8o anos, registrando 34,98\% ( $\mathrm{n}=205$ casos). Podemos ver neste estudo que a septicemia acomete principalmente a população da terceira idade, caracterizando um grave problema de saúde pública.

De acordo com o estudo transversal de Fonseca (2019), realizado com base nas notificações do CID1o - septicemia, nas regiões do Brasil, pode-se destacar que os pacientes com maior grau de acometimento pela infecção é o dos pacientes de 60 a 8 o anos ou mais representam 55,33\% da amostra. Corroborando com a atual pesquisa aqui elaborada. Ainda sobre o estudo de Fonseca (2019), na Região Nordeste o total de casos de pacientes acometidos pela sepse, foram de $20,24 \%$, se comparado ao total.

Outro trabalho que corrobora com a atual pesquisa, é a de Cesaro e Zonta (2018), realizado no Hospital Público do Paraná, no período de janeiro de 2012 à janeiro de 2017, que dos 1.557 prontuários analisados, 345 (62,3\%) eram do sexo masculino e 209 (37,7\%) eram do sexo feminino, com idade superior a setenta anos $(25,8 \%)$.

Staphylococcus aureus causa abscessos, várias infecções piogénicas (p. ex., endocardite, artrite séptica e osteomielite), intoxicação alimentar, síndrome da pele escaldada e síndrome do choque tóxico. $\mathrm{O}$ organismo é uma das causas mais comuns de pneumonia 
hospitalar, septicemia, e infecções de feridas cirúrgicas. É uma importante causa de infecções cutâneas, como foliculite, celulite e impetigo. Staphylococcus epidermidis pode causar endocardite e infecções em articulações prostéticas. Staphylococcus saprophyticus causa infecções do trato urinário. A síndrome de Kawasaki é uma doença de etiologia desconhecida, que pode ser causada por determinadas linhagens de S. aureus. (LEVINSSON, p. 113, 2011).

Os idosos são mais acometidos por sepse, de acordo com Santos et al. (2019), muitos vão a óbitos pelas complicações acarretadas pela doença, pois é na terceira idade que as pessoas estão mais suscetíveis a infecções oportunistas. Ainda de acordo com a Organização Mundial de Saúde, pacientes com idade maior que 65 anos, estão sujeitos a sepse e choque séptico, ocasionados por outras doenças crônicas, corpo em desequilíbrio funcional e sistema imune incapaz de deter processos infecciosos mais complexos.

De acordo com a seguinte variável, óbitos de acordo com ano e mês, segundo o sexo dos pacientes, destacamos os seguintes dados ilustrados na tabela número 2.

Tabela 2. Óbitos de acordo com ano e mês, segundo o sexo dos pacientes.

\begin{tabular}{lccc}
\hline Mês/Ano & Sexo Masculino & Sexo Feminino & Total \\
\hline 2019/Jul. & 39 & 42 & 81 \\
2019/Ago. & 17 & 26 & 43 \\
2019/Set. & 31 & 25 & 56 \\
2019/Out. & 32 & 30 & 62 \\
2019/Nov. & 33 & 31 & 64 \\
2019/Dez. & 23 & 22 & 45 \\
2020/Jan. & 24 & 35 & 59 \\
2020/Fev. & 27 & 26 & 53 \\
2020/Mar. & 29 & 32 & 61 \\
2020/Abr. & 34 & 28 & 62 \\
\hline Total & 289 & 297 & 586 \\
\hline
\end{tabular}

Fonte: DATASUS, 2020.

Podemos ver que a maior taxa de mortalidade ocorreu no sexo feminino, representando um percentual de 50,42\% ( $\mathrm{n}=297$ casos), enquanto que no gênero masculino o percentual foi de 49,31\% ( $\mathrm{n}=289$ casos).

Consoante Magolin et al. (2018), em seu estudo observacional, transversal e retrospectivo, desenvolvido em hospital particular de Niterói-RJ, observou-se que os pacientes com suspeita de sepse, segundo o gênero sexual, representavam sendo 55,8 
$\%(n=115)$ do sexo feminino e $44,2 \%(n=91)$ do sexo masculino, com idade media de 62,51 anos.

Com base no estudo epidemiológico de Santos et al. (2019), dos 7.764 casos de internações em pacientes com sepse, no período de 2012 a 2017, podemos ver que o percentual mais acometido pela infecção foi no gênero masculino percentuando um total de $53,73 \%$ ( $n=4.172$ casos) e quanto ao sexo feminino apresentou 43,26\% ( $n=3.592)$.

De acordo com o estudo epidemiológico de Volpáti et al. (2019), dos 23 (57,5\%) pacientes do sexo masculino, diagnosticado com sepse, apenas $12(52,2 \%)$ vieram a óbito, e $11(47,8 \%)$ receberam alta da unidade de Terapia Intensiva, enquanto que o sexo feminino dos $17(42.5 \%)$ apenas 7 vieram a óbito, e as 10 restantes receberam alta, neste estudo podemos observar que os homens foram mais afetados pela sepse.

Tabela 3. Óbitos por gênero, segundo capítulo do CID-10.

\begin{tabular}{lccc}
\hline Capitulo CID-1o & $\begin{array}{c}\text { Sexo } \\
\text { masculino }\end{array}$ & $\begin{array}{c}\text { Sexo } \\
\text { Feminino }\end{array}$ & Total \\
\hline I. Algumas doenças infecciosas e parasitárias & 617 & 602 & 1.219 \\
II. Neoplasias (Tumores) & 377 & 413 & 790 \\
III. Doenças sangue órgãos hemat. e transt. Imunitário & 33 & 28 & 61 \\
IV. Doenças endócrinas nutricionais e metabólicas & 75 & 98 & 173 \\
V. transtornos mentais e comportamentais & 8 & 3 & 11 \\
VI. Doenças do sistema nervoso & 44 & 49 & 93 \\
VII. Doenças dos olhos e anexos & 1 & - & 1 \\
VIII. Doenças do ouvido e da apófise mastoide & - & 1 & 1 \\
IX. Doenças do aparelho circulatório & 585 & 530 & 1.115 \\
X. Doenças do aparelho respiratório & 471 & 488 & 959 \\
XI. Doenças do aparelho digestivo & 285 & 191 & 476 \\
XII. Doenças da pele e do tecido subcutâneo & 45 & 47 & 92 \\
XIII. Doenças sist. Osteomuscular e tec. Conjuntivo & 3 & 1 & 4 \\
XIV. Doenças do aparelho geniturinário & - & 121 & 268 \\
XV. Gravidez parto e puerpério & 147 & 8 & 8 \\
XVI. Algumas afec. Originadas no período perinatal & 94 & 81 & 175 \\
XVII. Malf. Congênita, def. e anom. Cromossômicas & 6 & 5 & 11 \\
XVIII. Sint. Sinais e achad. Anorm. Ex clín. e laboratorial & 56 & 47 & 103 \\
XIX. Lesões enven. E alg. Out. conseq. Causas externas & 221 & 94 & 315 \\
XXI. Contatos com serviços de saúde & 8 & 9 & 17 \\
\hline Total & 3.076 & 2.816 & 5.892 \\
\hline
\end{tabular}

Fonte: DATASUS, 2020. 
A sepse entra no capitulo I, no qual diz respeito às Doenças infecciosas e parasitárias, neste estudo o número total foi de $20,68 \%$ ( $n=1.219$ casos), número que corresponde a homens e mulheres, sendo que desse total de óbitos, 48,07\% ( $n=586)$, foi ocasionado pela septicemia, no período de julho de 2019 a abril de 2020. Podemos também considerar que a sepse é um grande problemas para as Unidades de Terapia intensiva, por que representa um dos maiores causadores de obituários.

De acordo com o estudo de Coimbra et al. (2018), em sua pesquisa observacional, verificamos que a mortalidade por gênero segundo capitulo I do CID-10, o total foi de 41 casos, sendo 16 homens e 25 mulheres. Ainda sobre o estudo podemos destacar que a Sepse pulmonar que mais causou óbitos, percentuando um total de $34,14 \%$ ( $n=14$ casos) para homens, e 36,58\% ( $n=15$ casos) para mulheres, constatando-se que a Sepse é a segunda causa mais frequente de óbitos.

Outro estudo que corrobora este estudo é o de Soratto e Silva (2019), no qual os pacientes da Unidade de Terapia intensiva de um hospital de grande porte da Região do Extremo Sul Catarinense no período de Março de 2017 a Março de 2018, foram a óbito. Dos pacientes morreram, $48,9 \%$ foi por causa de doenças infecciosas parasitarias, nessa categoria foi destacada a sepse como maior responsável, pela metade de óbitos imediatos, nos pacientes.

\section{CONSIDERAÇÕES FINAIS}

O presente estudo enfatizou a importância de manter as autoridades epidemiológicas em alerta, quanto a sepse, uma condição que afeta maior parte dos pacientes nas, Unidade de Terapias Intensivas, causando maior taxa de mortalidade.

Com base nos dados apresentados pela presenta pesquisa, podemos ver as heterogeneidades das causas dos óbitos, afligem diferentes tipos de pessoas, nos atendimentos de saúde. $\mathrm{O}$ mapeamento do perfil epidemiológico da sepse é muito importante para que sejam criadas e adotadas novas estratégias para diminuir a Septicemia, reduzir a taxa de mortalidade, melhorar a saúde, ampliar a qualidade de vida, bem como a expectativa de vida das pessoas.

Os profissionais de saúde devem está sempre preparados e ter atenção no diagnostico precoce da sepse, pois como vimos neste estudo, a mesma tem potencial alto para a mortalidade humana, campanhas educativas e teóricas devem ser adotadas, com base no Ministério da Saúde, para adesão a tratamentos mais eficazes perante a infecção.

A Saúde pública deve está mapeando o obituário causado por sepse, afim de que os próximos estudos na área possam passar resultados confirmatórios e reais, para que então o profissional da saúde possa atuar com veemência nos estabelecimentos de saúde, prevenindo e tratando a sepse. 


\section{REFERENCIAS}

COIMBRA, Bruno Vasconcelos; GARCIA, Caroline Campos; GUERRA, Thais de Rezende Bessa. Perfil de causa mortis em idosos internados em um serviço público de urgência e emergência: evidências clínicas. Academus Revista Científica da Saúde, 2018, 3.2: 29-35.

da Fonseca, E. J. (2019). Análise epidemiológica da sepse no sistema público de saúdediferenças entre capitais e municípios brasileiros nos últimos 5 anos. Revista Ciências da Saúde e Educação IESGO, 1(2).

de Almeida, B. M., de Lima, R. B., \& da Silva, J. D. A. G. (2018). SEPSE EM QUEIMADOS ANÁLISE DE INCIDÊNCIA E MORTALIDADE DA SEPSE EM PACIENTES INTERNADOS NA UNIDADE DE TRATAMENTO DE QUEIMADOS DO HOSPITAL REGIONAL DA ASA NORTE. Programa de Iniciação Científica-PIC/UniCEUBRelatórios de Pesquisa, 3(1).

DE CESARO, Maiara Cristina;ZONTA, Francieledo NascimentoSantos. Epidemiologia de pacientes de uma UTI em um hospital público do Paraná que desenvovleram sepse comunitária/Epidemiology of patients of a UTI in a public hospital of Paraná who developed community sepse. Brazilian Journal of Health Review, 2018, 2.1: 501-506.

Jost, M. T., Machado, K. P. M., De Oliveira, A. P. A., da Costa Linch, G. F., Paz, A. A., Caregnato, R. C. A., \& Blatt, C. R. (2019). Morbimortalidade e custo por internação dos pacientes com sepse no Brasil, Rio Grande do Sul e Porto Alegre.

LEVISON W. Microbiologia medica e imunologia. $10^{\circ}$ ed., Porto Alegre, PA: Artmed, 2011.

Luz Filho, C. A., \& Marinho, C. M. M. (2019). Fatores de risco em pacientes com sepse em unidades de terapia intensiva: uma revisão integrativa. Revista Eletrônica Acervo Saúde, (19), e2o8-ezo8.

MANGOlin A., ANGIOLI COSTA W. M. M. D., AVELAR J. G., MARQUES E. A., ALVES Y., TEIXEIRA M. F., HORTALA JUNIOR C. C., ALMEIDA M. R. A. Perfil epidemiológico da sepse no departamento de emergência. Revista brasileira de terapia intensiva. São Paulo. 2018.

Menezes, L. E. F. J., de Negreiros, L. M. V., Maciel, L. B. C., Marques, T. A., Roballo, C. A., \& Baffa, A. M. (2019). Perfil epidemiológico e análise da efetividade para prevenção de óbitos de pacientes inseridos em protocolo de sepse. Revista da Sociedade Brasileira de Clínica Médica, 17(1), 25-30. 
Patricio, K. C., \& Domingues, G. A. (2019). Mortalidade intra-hospitalar e perfil epidemiológico de pacientes com cirrose hepática internados em um hospital de referência no extremo sul catarinense.

Ribeiro, M.S., Pires, H. F. M. (2018). SEPSE EM UNIDADE DE TERAPIA INTENSIVA EM UM HOSPITAL PÚBLICO: ESTUDO DA PREVALÊNCIA, CRITÉRIOS DIAGNÓSTICOS, FATORES DE RISCO E MORTALIDADE. Programa de Iniciação Científica-PIC/UniCEUB-Relatórios de Pesquisa, 3(1).

Rocha, N. P. M., Maneschy, R. B., Xavier, L. S. M., Xavier, L. C., Pontes, C. D. N., de Holanda, L. S., ... \& Santos, A. M. (2019). Impacto sobre a evolução de pacientes sépticos após implementação de um protocolo institucional de sepse em um hospital público em Belém-PA. Revista Eletrônica Acervo Saúde, 11(3), e255-e255.

Santos, A. F. S., da Silva, I. B., Calheiros, T. R. S. P., Júnior, A. F. S. X., Tenório, J. O. C., da Rocha, D. M., ... \& de Lima Souza, W. (2019). PERFIL DAS AUTORIZAÇÕES DE INTERNAÇÃO HOSPITALAR POR SEPSE NO PERÍODO DE 2012 A 2017 EM ALAGOAS, BRASIL. Revista de Pesquisa em Saúde, 19(2).

Santos, J. V. V. R., dos Santos, R. W. F., dos Santos, R. C. F., Barreto, L. S., dos Santos, R. M., \& Rodrigues, I. D. S. V. (2018). O USO DE ANTIMICROBIANOS EM NEONATOS DIAGNOSTICADOS COM SEPSE. Semana de Pesquisa da Universidade TiradentesSEMPESq, (19).

Seibt, E. T., Kuchler, J. C., \& Zonta, F. D. N. S. (2019). Incidência e características da sepse em uma unidade de terapia intensiva de um hospital misto do Paraná. Revista de Saúde Pública do Paraná, 2(2), 97-106.

Silva, L. M., Bicalho, E. A. G., \& Caixeta, A. C. M. (2018). INCIDÊNCIA DE ÓBITOS POR SEPSE EM NEONATOS. Psicologia e Saúde em debate, 4(3), 20-31.

Soratto, B. M., \& Silva, L. F. D. (2019). Perfil dos óbitos na unidade de terapia intensiva de um hospital de grande porte do extremo sul catarinense.

TORTORA G. J., FUNKE B.R., CASE C. L. Microbiologia. 10 ed., Porto Alegre, PA: Artmed, 2012.

VOLPÁTI N. V., PRADO P. R., MAGGI L. E. Perfil epidemiológico dos pacientes com sepse de foco abdominal. Rev enferm UFPE on line. 2019

WEBER, G. (2019). Série de casos de óbitos de pacientes em decorrência de sepse em uma Unidade de Terapia Intensiva no nordeste brasileiro. 
Zonta, F. N. S., Velasquez, P. G. A., Velasquez, L. G., Demetrio, L. S., Miranda, D., \& Silva, M. C. B. D. (2018). Epidemiological and clinical characteristics of sepse in a public hospital of Paraná. 